\title{
High pulse energy, picosecond MgO:PPLN optical parametric oscillator using a single-mode fiber for signal feedback
}

\author{
Florian Kienle ${ }^{1, *}$, Peh S. Teh ${ }^{1}$, Shaif-Ul Alam ${ }^{1}$, Corin B. E. Gawith ${ }^{2}$, David C. Hanna ${ }^{1}$, David J. Richardson ${ }^{1}$, \\ David P. Shepherd ${ }^{1}$ \\ ${ }^{1}$ Optoelectronics Research Centre, University of Southampton, Highfield, Southampton, SO17 1BJ, United Kingdom \\ ${ }^{2}$ Covesion Ltd., Romsey, SO51 9AQ, United Kingdom \\ *Corresponding author: flk@orc.soton.ac.uk
}

\begin{abstract}
We demonstrate a high-pulse-energy, synchronously-pumped (7.19MHz), 100ps, widely tunable MgO:PPLN OPO providing $0.49 \mu \mathrm{J}$ pulses at $1.5 \mu \mathrm{m}$ and $0.19 \mu \mathrm{J}$ pulses at $3.6 \mu \mathrm{m}$. A single-mode fiber is employed in the OPO to keep the $42 \mathrm{~m}$-long cavity compact. OCIS codes: $190.0190,190.4970,140.7090,060.2320,140.2020$
\end{abstract}

\section{Introduction}

Synchronously-pumped optical parametric oscillators (SPOPO) are useful sources for ultrashort, tunable optical pulses. Progress in high-power, compact, ultrafast pump sources means that they are becoming ever more attractive for a broad range of applications. Advances have included a high-average-power femtosecond OPO pumped by a thin-disk Yb:YAG laser [1], a high-pulse-energy picosecond OPO pumped by an Yb:fiber laser [2], and a controllable repetition rate picosecond OPO pumped by a fiber-amplified gain-switched laser diode [3].

In this contribution, we report a picosecond SPOPO outputting high-energy pulses by using a pump source consisting of a 100ps gain-switched laser diode operating at $7.19 \mathrm{MHz}$ and amplified in three Yb-doped fiber amplifiers, similar to that in [4], and by applying a single-mode fiber in the SPOPO cavity for signal feedback [1] to maintain a compact device.

\section{Experimental setup}

The $1.06 \mu \mathrm{m}$ pump source was similar in all respects to that described previously [4]. However, the chirped fiber Bragg grating, used to compensate for the predominantly linear chirp developed on the gain-switched seed pulse, was by-passed here leading to an increase of the pulse duration from 20ps to 100ps. The reduced peak power helped to avoid nonlinear effects in the fiber amplifiers enabling operation with pulse energies of up to $8.6 \mu \mathrm{J}$, corresponding to an average power of $62 \mathrm{~W}$ at the $7.19 \mathrm{MHz}$ repetition rate. The bandwidth of the output pulses was $0.25 \mathrm{~nm}$ and was thus $\sim 10$ times the bandwidth limit. This highly efficient and compact pump source delivered neardiffraction-limited $\left(\mathrm{M}^{2} \leq 1.2\right.$ at any power level), linearly polarized output pulses with user-controllable repetition rate and a minimum of free-space optical components.

A schematic diagram of the SPOPO system is shown schematically in figure 1 . The majority of the optical path length of the SPOPO ring cavity was provided by a $\sim 27 \mathrm{~m}$ piece of standard single-mode fiber (Corning SMF28). This allowed a small-footprint and compact overall resonator layout. A periodically-poled, 40x10x1 $\mathrm{mm}^{3}$ MgO: $\mathrm{LiNbO}_{3}$ (MgO:PPLN) crystal was used as gain medium (Covesion Ltd.), where the 40mm length provided sufficient phase-matching bandwidth and relatively small temporal walk-off for the 100ps pump and signal pulses. The crystal had seven, $1 \mathrm{~mm}$ by $1 \mathrm{~mm}$, periodically-poled gratings with periods ranging from $29.5 \mu \mathrm{m}$ up to $31.5 \mu \mathrm{m}$, and was held in an oven at $150^{\circ} \mathrm{C}$ to eliminate any residual photorefractive effects. By using a lens with $250 \mathrm{~mm}$ focal length to focus the pump beam into the crystal, a spot radius $\left(1 / \mathrm{e}^{2}\right.$-intensity) of $104 \mu \mathrm{m}$ was achieved giving a good match to the calculated $99 \mu \mathrm{m}$ signal spot radius obtained with $250 \mathrm{~mm}$ radius of curvature mirrors CM1 and CM2.

A $90 \%$ transmission output coupler was used in order to achieve efficient output power extraction. The high output coupling also ensured that relatively low signal powers were returned through the optical fiber in order to avoid nonlinear distortions such as Raman scattering. Nevertheless, a fiber-coupling arrangement with a heat-sink was required to avoid gradual damage to the fiber end-facet. The MgO:PPLN crystal and the coupling lenses L1, L2 were broad-band anti-reflection coated at the signal wavelengths, and the MgO:PPLN coating was also low reflectivity for the pump and idler wavelengths. The cavity was singly-resonant for the signal with CM1 and CM2 being highly reflective for the signal wavelengths and all cavity mirrors being highly transmissive for the idler wavelengths. Furthermore, the fiber was not transmissive at mid-infrared wavelengths. The idler was extracted 
through CM2, which had a transmission of $88 \%$ at the idler wavelengths. The transmission of CM1 for the pump wavelength of $1.06 \mu \mathrm{m}$ was $92 \%$.

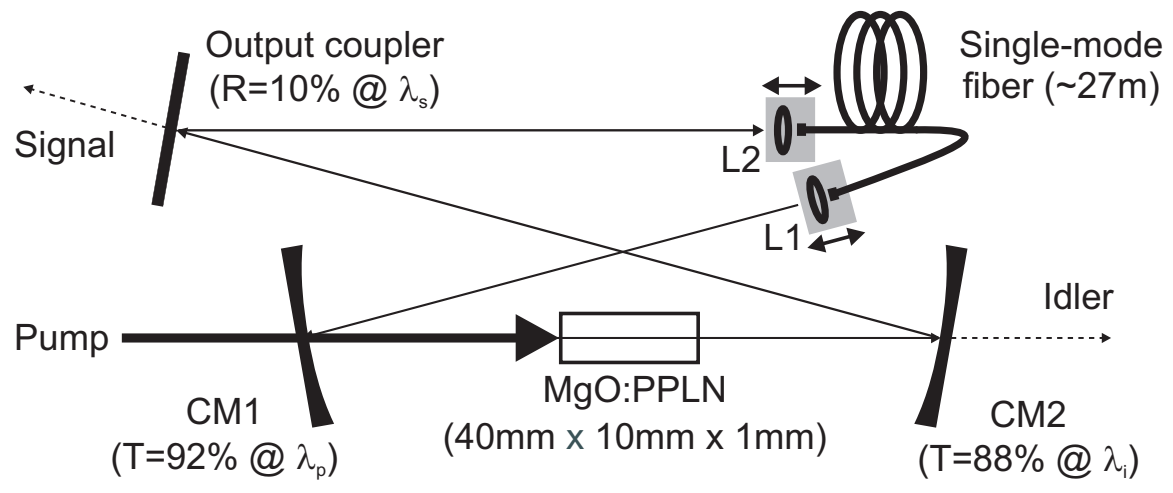

Fig. 1. Schematic diagram of the SPOPO fiber feedback cavity.

\section{Results}

Figure 2 shows the signal and the idler output power of the SPOPO as a function of the input pump power by using the $T=90 \%$ signal output coupler. All power values given here are measured external to the cavity. The oscillation threshold was achieved at an average pump power of $3.94 \mathrm{~W}$. Up to $3.51 \mathrm{~W}$ of average signal power at $1.504 \mu \mathrm{m}$ and $1.37 \mathrm{~W}$ of average idler power at $3.591 \mu \mathrm{m}$ was obtained using the $29.5 \mu \mathrm{m}$-period grating and average pump powers up to $20.07 \mathrm{~W}$. The pump depletion was typically $35 \%$. These values correspond to pulse energies of $0.49 \mu \mathrm{J}$ and $0.19 \mu \mathrm{J}$ for the signal and idler, respectively. The highest pulse energy of $0.65 \mu \mathrm{J}$ (signal only, idler not used) reported previously stems from a relay-imaging free-space OPO resonator by employing a cavity dumping technique [5].

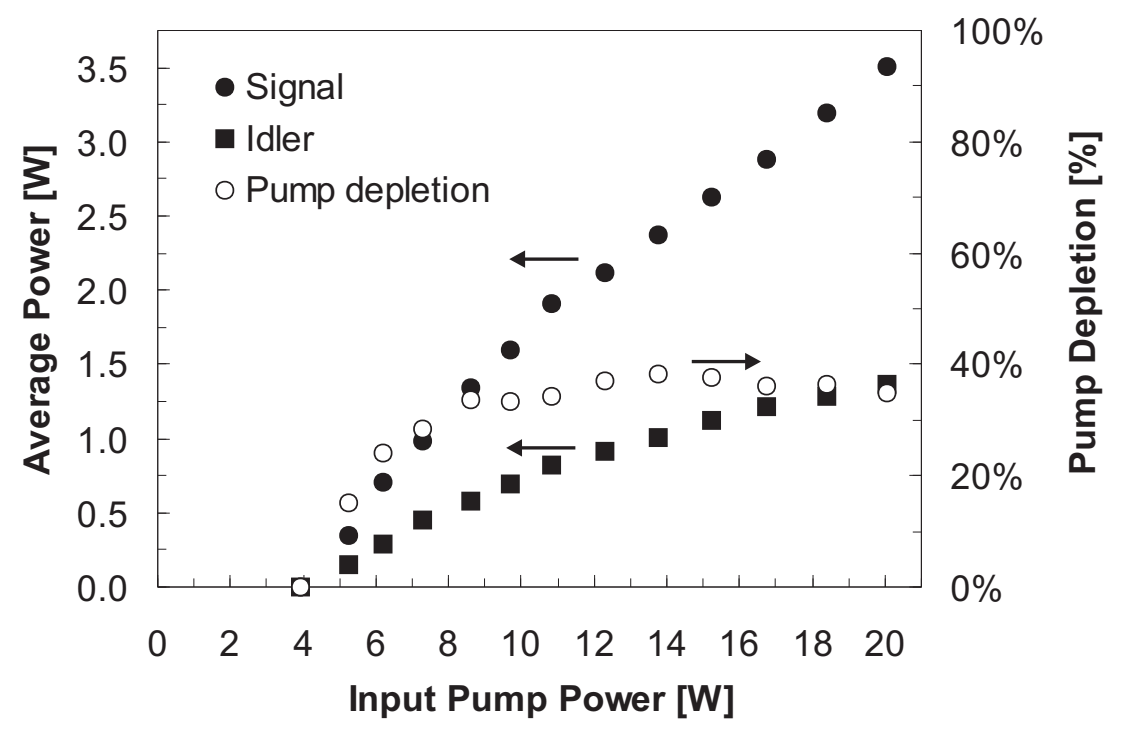

Fig. 2. Signal and idler output power as a function of input power. Pump depletion is shown on the right.

A manual fiber polarization controller was used in an attempt to control the polarization of the non-polarizationmaintaining single-mode fiber and to deliver a linearly polarized beam with the correct orientation to the MgO:PPLN crystal. However, it was observed experimentally that this had no effect on the OPO output power. The parametric gain was in the high gain regime, where we estimated a required gain at the oscillation threshold of $\sim 40$ to overcome the losses due to the high output coupler transmission, the loss at fiber launching and the loss due to the incorrect polarization of the beam at the fiber output. In this regime the gain is given by $1 / 4 \cdot \exp (2 \Gamma \mathrm{L}), \mathrm{where} \mathrm{L}$ is the crystal length and $\Gamma$ is the gain coefficient, as defined in [6]. Hence, the threshold gain exponent $2 \Gamma \mathrm{L}$ was $\sim 5$. At the maximum pump power, $\sim 5$ times above threshold, the exponent is $\sqrt{5}$ times greater (because $\Gamma^{2}$ is proportional to the pump power), i.e. $2 \Gamma \mathrm{L}=11.2$, corresponding to a gain of $\sim 43 \mathrm{~dB}$. An accurate $a b$ initio calculation of the 
effective gain exponent is complicated by the uncertainty over the effective pump intensity, since it has a Gaussian rather than a plane wave form.

We observed instability and a power roll-over of the output power when increasing the average pump power beyond 20W. The reason for the roll-over is not entirely clear but it appears to be not related to average power limitations for the MgO:PPLN crystal as a reduction of the pump average power and hence the thermal input to the crystal by placing a 50/50 duty cycle optical chopper in the pump beam did not improve the performance of the SPOPO. Physical damage, e.g. cracking or coating damage, of the crystal was not observed. There are indications that the limitation may lie in the pump source, especially in the optical isolator used between pump source and SPOPO. The crystal of the isolator may suffer from thermal effects, leading to a beam quality degradation and/or a spatial beam drift. Future work will investigate these limitations further.

The signal beam duration was measured with a $32 \mathrm{GHz}$ InGaAs detector and a $50 \mathrm{GHz}$ communication signal analyzer (experimentally determined minimum measurable pulse duration of $\sim 30 \mathrm{ps}$ ) to be 100ps and thus similar to that of the pump. Spectra were taken for both the signal (with an optical spectrum analyzer) and idler (with a monochromator) pulses giving FWHM values of $2.0 \mathrm{~nm}$ and $12 \mathrm{~nm}$, respectively. These figures indicate strongly chirped pulses, which is again similar to the performance of the pump laser. The SPOPO signal and idler wavelength was tuned by accessing different poled gratings of the MgO:PPLN with the pump beam and a corresponding cavity length adjustment to account for the changing round-trip time of the signal pulses due to dispersion. A signal tuning range from $1.5 \mu \mathrm{m}$ to $1.7 \mu \mathrm{m}$ and an idler tuning range from $2.9 \mu \mathrm{m}$ to $3.6 \mu \mathrm{m}$ was achieved. The $M^{2}$-values were determined at the highest power to be 1.5 by 1.3 for the signal and 2.8 by 1.9 for the idler, where the higher value for both beams corresponds to the axis perpendicular to the nonlinear crystal plane and parallel to the ring cavity plane.

\section{Summary}

In summary, we have demonstrated a high pulse energy, synchronously-pumped, picosecond SPOPO operating at low $\mathrm{MHz}$ repetition rate. The use of a single-mode fiber to provide signal feedback is a key feature to maintain a compact resonator size despite its long cavity length. In combination with the efficient, compact, and power-scalable pump source consisting of a gain-switched laser diode and a chain of three Yb-doped fiber amplifiers, the overall system is attractive as a potential source for infrared materials processing $[7,8]$. Future work will concentrate on further power scaling into the $\mu \mathrm{J}$ pulse energy regime at pulse durations of a few picoseconds.

\section{References}

[1] T. Südmeyer, E. Innerhofer, F. Brunner, R. Paschotta, T. Usami, H. Ito, S. Kurimura, K. Kitamura, D. C. Hanna, and U. Keller, "High-power femtosecond fiber-feedback optical parametric oscillator based on periodically poled stoichiometric LiTaO3” Opt. Lett. 29, 1111-1113 (2004).

[2] T. P. Lamour, L. Kornaszewski, J. H. Sun, and D. T. Reid, "Yb:fiber-laser-pumped high-energy picosecond optical parametric oscillator," Opt. Express 17, 14229-14234 (2009).

[3] F. Kienle, K. K. Chen, S.-U. Alam, C. B. E. Gawith, J. I. Mackenzie, D. C. Hanna, D. J. Richardson, and D. P. Shepherd, "High-power, variable repetition rate, picosecond optical parametric oscillator pumped by an amplified gain-switched diode," Opt. Express 18, 7602-7610 (2010).

[4] K. K. Chen, J. H. V. Price, S.-U. Alam, J. R. Hayes, D. Lin, A. Malinowski, D. J. Richardson, "Polarisation maintaining 100W Yb-fiber MOPA producing $\mu \mathrm{J}$ pulses tunable in duration from 1 to $21 \mathrm{ps,"} \mathrm{Opt.} \mathrm{Express} \mathrm{18,} \mathrm{14385-14394} \mathrm{(2010).}$

[5] T. P. Lamour, J. H. Sun, and D. T. Reid, "High-pulse-energy cavity-dumped optical parametric oscillator in the near- and mid-infrared," presented at the International Summer School in Ultrafast Nonlinear Optics, SUSSP 66, Edinburgh, Scotland, 11-21 August 2010.

[6] R. L. Byer and R. L. Herbst, "Parametric Mixing and Oscillation,” in Nonlinear Infrared Generation, Y. R. Shen ed. (Springer, Berlin, 1977), pp. 81-137.

[7] D. M. Bubb and R. F. Haglund Jr., "Resonant Infrared Pulsed Laser Ablation and Deposition of Thin Polymer Films," in Pulsed Laser Deposition of Thin Films, R. W. Eason ed. (John Wiley \& Sons, 2006), pp. 35-60.

[8] V. Z. Kolev, M. W. Duering, B. Luther-Davies, and A. V. Rode, “Compact high-power optical source for resonant infrared pulsed laser ablation and deposition of polymer materials," Opt. Express 14, 12302-12309 (2006). 